\title{
Spatial patterns of Plasmodium vivax transmission explored by multivariate auto-regressive state-space modelling - A case study in Baoshan Prefecture in southern China
}

\author{
Jinxin Zheng, ${ }^{1-4}$ Benyun Shi, ${ }^{5}$ Shang Xia, ${ }^{1-4}$ Guojing Yang, ${ }^{6-8}$ Xiao-Nong Zhou ${ }^{1-4}$ \\ ${ }^{1}$ National Institute of Parasitic Diseases, Chinese Center for Disease Control and Prevention, Shanghai, \\ China; ${ }^{2}$ Key Laboratory of Parasite and Vector Biology, National Health Commission, Shanghai, China; \\ ${ }^{3}$ National Center for International Research on Tropical Diseases, Ministry of Science and Technology, \\ Shanghai, China; ${ }^{4}$ Chinese Center for Tropical Diseases Research, Shanghai, China; ${ }^{5}$ School of Computer \\ Science and Technology, Nanjing Tech University, Nanjing, Jiangsu, China; ${ }^{6}$ Hainan Medical University, \\ Laboratory of Tropical Environment and Health, Haikou, Hainan, China; ${ }^{7}$ Department of Epidemiology \\ and Public Health, Swiss Tropical and Public Health Institute; ${ }^{8}$ University of Basel, Basel, Switzerland
}

\begin{abstract}
The transition from the control phase to elimination of malaria in China through the national malaria elimination programme has focussed attention on the need for improvement of the surveillance-response systems. It is now understood that routine passive
\end{abstract}

Correspondence: Xiao-Nong Zhou, National Institute of Parasitic Diseases, Chinese Center for Disease Control and Prevention, Shanghai 200025, China. E-mail: zhouxn1@chinacdc.cn

Key words: Malaria; Plasmodium vivax; indigenous case; state space model; foci; cluster; China.

Acknowledgements: we would like to thank the support of Disease Prevention and Control Center of Yunnan Province, China, and Chinese Center for Disease Control and Prevention in Shanghai and all the staff members in data collection and investigation. We are also grateful to the study participants for their commitment.

Funding: this research was funded by National Natural Science Foundation of China (Grant No. 81402760, 81573261, 81502858), as well as a grant from Natural Science Foundation of Jiangsu Province, China (Grant No. BK20161563) also with the Open Project of Key Laboratory of Parasite and Vector Biology, China Ministry of Health (WSBKFKT-201804). The funders had no role in study design, data collection and analysis, decision to publish, or preparation of the manuscript.

Conflict of interest: the authors declare no potential conflict interests.

Received for publication: 9 March 2020.

Revision received: 21 August 2020.

Accepted for publication: 21 August 2020.

(C) Copyright: the Author(s), 2021

Licensee PAGEPress, Italy

Geospatial Health 2021; 16:879

doi:10.4081/gh.2021.879

This article is distributed under the terms of the Creative Commons Attribution Noncommercial License (CC BY-NC 4.0) which permits any noncommercial use, distribution, and reproduction in any medium, provided the original author(s) and source are credited. surveillance is inadequate in the parasite elimination phase that requires supplementation by active surveillance in foci where cluster cases have occurred. This study aims to explore the spatial clusters and temporal trends of malaria cases by the multivariate auto-regressive state-space model (MARSS) along the border to Myanmar in southern China. Data for indigenous cases spanning the period from 2007 to 2010 were extracted from the China's Infectious Diseases Information Reporting Management System (IDIRMS). The best MARSS model indicated that malaria transmission in the study area during 36 months could be grouped into three clusters. The estimation of malaria transmission patterns showed a downward trend across all clusters. The proposed methodology used in this study offers a simple and rapid, yet effective way to categorize patterns of foci which provide assistance for active monitoring of malaria in the elimination phase.

\section{Introduction}

Malaria is a life-threatening disease caused by Plasmodium spp. parasites that are transmitted through the bite of infected female Anopheles spp. mosquitoes. In 2019, the World Health Organization (WHO) estimated the worldwide disease burden at nearly 228 million cases and 405,000 deaths (WHO, 2019). In order to control and eliminate malaria globally, various projects or programmes have been proposed, such as the Global Malaria Programme (Whittaker et al., 2014), the Asia Malaria Elimination Network (Gosling et al., 2012), and the Strategy for Malaria Elimination in the Greater Mekong Sub-region (Cui et al., 2012) and others. Ten years ago, the National Malaria Elimination Action Plan (NMEAP) was launched by the Ministry of Health $(\mathrm{MoH})$ in the People's Republic of China (MoH, 2010). Thanks to effective strategies implemented by the Chinese Government, the malaria incidence rate has decreased significantly since then. NMEAP included disease management and vector control, which have been confirmed to be powerful and co-effectiveness measures (Yin et al., 2014; Zhou et al., 2015b; Hu et al., 2016).

Most malaria cases are reported from south-western China, especially along the China-Myanmar border in Yunnan Province (Wang et al., 2014; Shi et al., 2015; Xu et al., 2016). Indeed, from 2013 to 2017, up to $90 \%$ of all indigenous malaria cases in China originated in these areas (Zhou et al., 2011; Li et al., 2016; Feng 
et al., 2018; Zhang et al., 2018). For examples, 3078 malaria cases were reported across the country in $2014,2 \%$ of which were indigenous and had occurred in the China-Myanmar border areas (Li et al., 2016). Thus, malaria transmission here remains a big challenge for NMEAP, which aims to free the country of malaria by 2020 .

Active surveillance is an effective approach in the malaria elimination phase (Zhang et al., 2017; Zhou et al., 2015b). However, implementation of this kind of surveillance in the endemic areas is a crucial matter, especially in the areas characterized by complicated environmental settings. The geographic variations of malaria transmission in China-Myanmar border areas have shown an underlying spatial pattern of malaria incidence providing a reference to design local surveillance strategies (Shi et al., 2015). Along with this point, it is necessary to explore the spatial patterns of malaria transmission so as to provide evidence-based data that can improve the sensitivity of the surveillance-response system in the elimination phase. Since 2004, the Infectious Diseases Information Reporting Management System (IDIRMS), established by the Chinese Center for Disease Control and Prevention (China CDC), different infectious diseases have been closely examined and scrutinized in the county health care institutions and then rapidly reported to IDIRMS. This well-run data collection provides a good opportunity to analyze the dynamic patterns of malaria transmission, e.g., by mathematic modelling, which has provided a way to quickly estimate or predict transmission risks of infectious diseases.

Ever since the availability of large datasets including times series on disease transmission, the auto-regressive integrated moving average (ARIMA) approach has been widely used to forecast the transmission pattens of vector-borne diseases (Wangdi et al., 2010; Anwar et al., 2016). These studies mainly focused on single time series (Wangdi et al., 2010) rather than multiple ones from different sites. However, studies in this field have started to apply techniques, so far mainly used in artificial intelligence and game theory, for analytical investigations of multivariate time-series in fields, such as parasitology, ecology, economics and genetics (Valle and Clark, 2013; Bartocci and Lió, 2016; Wang, He et al., 2016; Tolimieri et al., 2017). The state-space is central in this kind of modelling. It represents all possible configurations of a system, where the coordinates are state variables that together describes the state of the system completely, which means that each point in the state-space corresponds to a different state of the system. As this kind of modelling uses abundant time series to balance limited data support, this strategy is suitable for dealing with small areas and populations. The multivariate auto-regressive state-space (MARSS) technique, originally proposed by Hinrichsen (2009) and further developed by Ward et al. (2010) and Holmes et al. (2012), provide a useful mathematical approach to work on multiple time series. Since this approach has also been applied to predict malaria transmission (Valle and Clark, 2013), we felt that it would be possible to attempt using MARSS for tracing malaria transmission in southern China. Baoshan, Yunnan Province, is located in south-western China near the China-Myanmar border. Due to the nature of the geological environment and mosquito species as well as frequent mobility of migrated population along the border, malaria is still a serious problem in this region. It has consistently shown the highest number of malaria cases due to Plasmodium vivax in the whole country during 2007-2013 (Xu et al., 2016). In this study, we attempted to develop a model providing a reference for decision-making with regard to surveillance policy aimed at eliminating malaria in China-Myanmar border areas by addressing two questions: i) is active surveillance of malaria in the Baoshan region consistent with the strategies applied in the geographical administrative regions and the disease foci?; and ii) do the Baoshan counties represent different spatial transmission patterns than seen elsewhere?

\section{Materials and methods}

\section{Study area}

Malaria transmission was studied in Baoshan, one of the prefectures in Yunnan Province bordering Myanmar, which has an area of $19,637 \mathrm{~km}^{2}$ and a population of 2.5 million based on the 2010 census. It has a tropical climate with abundant precipitation, averaging 700-1200 $\mathrm{mm}$ per year and a seasonal temperature fluctuation with peaks in May-August. There are five administrative counties under Baoshan, namely Changning, Longling, Longyang, Shidian and Tengchong (Figure 1). Malaria cases appear all year around in Baoshan Prefecture accounting for the majority of malaria cases in Yunnan Province and China as a whole before 2010, especially in Tengchong County near the China-Myanmar border with high numbers of annual malaria cases, the highest in China.

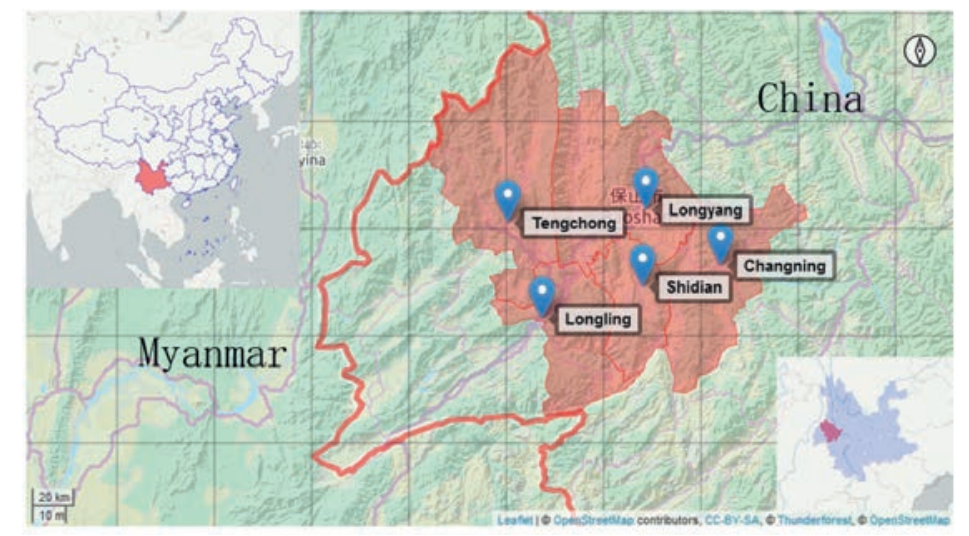

Figure 1. Map of malaria transmission in five counties in Baoshan Prefecture, Yunnan Province, China near Myanmar border. 


\section{Data collection}

Information on indigenous $P$. vivax cases in the study area were provided by IDIRMS of China CDC. We aggregated indigenous $P$. vivax incidence data from January 2007 to December 2009 by patient residential addresses, which were distributed over all five counties of Baoshan Prefecture. To fit the MARSS model, we used time series data transformed into the monthly distribution of $P$. vivax cases.

\section{State-space model}

The utilized the MARSS model as specified by Holmes et al. (2012), by which the total variance in the observation of time series is defined by partition into observation variance and process variance. In our study, the latter is the temporal variance of the malaria declining rate, while former includes sampling error and measurement error from data inaccurcies. The MARSS framework is given by two equations:

$$
\begin{aligned}
& X_{t}=B_{t} X_{t-1}+u_{t}+w_{t}, w_{t} \sim N\left[0, Q_{t}\right] \\
& Y_{t}=Z_{t} X_{t}+a_{t}+v_{t}, \quad v_{t} \sim N\left[0, R_{t}\right]
\end{aligned}
$$

We used $n$ to denote the number of discrete survey counties, and $m$ to represent the number of unknown hidden states (foci clusters). For the hidden states in Eq (1), $X_{t}$ is a matrix that denotes the vector of length $m$ representing the true observation indigenous cases in year $t$; $B_{t}$ a $m \times m$ matrix that allows the density dependence; $u_{t}$ a $m \times 1$ vector of hidden states of the declining rates; and $w_{t}$ a vector of length $m$ representing the process errors in year $t$. We assumed that the process errors can be correlated and specified from a multivariate normal distribution with a zero mean of a $m \times m$ matrix $Q_{t}$. In Eq (2), $Y_{t}$ is the $n$ element vector of observed malaria cases at time $t, Z_{t}$ a $n \times m$ matrix that defines $n$ observations related to the $m$ hidden states. In general, the $n$ sites could be any samples from the $m$ hidden sates. The $n \times 1$ vector specifies the bias between those sites. The observation errors at time $t$ are denoted by $v_{t}$, the same approach to address process error in Eq (1), which is an $n$ vector of serially uncorrelated disturbances with zero mean of an $n \times n$ matrix $R_{t}$. When using our data of all malaria cases from the five counties (the observed time series), we wanted to fit a model with one hidden state. All observed time series were sampled from this unique focus cluster. There was one process error variance with different observation variance. The observed malaria cases were independent of each other. Mathematically the fitted MARSS model is described by the following matrices:

$$
\begin{aligned}
& x_{t}=x_{t-1}+u+w_{t}, w_{t} \sim N(0, q) \\
& {\left[\begin{array}{l}
y_{1, t} \\
y_{2, t} \\
y_{3, t} \\
y_{4, t} \\
y_{5, t}
\end{array}\right]=\left[\begin{array}{l}
1 \\
1 \\
1 \\
1 \\
1
\end{array}\right] x_{t}+\left[\begin{array}{l}
0 \\
a_{2} \\
a_{3} \\
a_{4} \\
a_{5}
\end{array}\right]+\left[\begin{array}{l}
v_{1, t} \\
v_{2, t} \\
v_{3, t} \\
v_{4, t} \\
v_{5, t}
\end{array}\right], v_{t} \sim \operatorname{MVN}\left(0,\left[\begin{array}{ccccc}
r_{1} & 0 & 0 & 0 & 0 \\
0 & r_{2} & 0 & 0 & 0 \\
0 & 0 & r_{3} & 0 & 0 \\
0 & 0 & 0 & r_{4} & 0 \\
0 & 0 & 0 & 0 & r_{5}
\end{array}\right]\right)}
\end{aligned}
$$

In this formula, the $v_{t}$ comes from a multivariate normal distribution (MVN) with variance-covariance matrix.

\section{Model estimation and selection}

Our goal was to find all possible foci clusters in the dataset, then evaluate the best fitted model. Assuming that the observation errors are independent within all the sites, we fitted a series of models for all of sites in Baoshan, for each model the process error and reduction rates were set differently within each cluster. Our model selection was based on the value of Akaike's information criterion (AIC) as given by (Koc and Bozdogan, 2015). It offers an estimation of the relative information lost when a given model is used, and when the dataset is small, only a small sample modification can be supported (Holmes et al., 2012). We used the corrected AIC (AICc) that is adjusted to small datasets. The maximum likelihood parameter is used to provide fully parametric bootstraps, when the outputs of parameters are sampled from a numerically estimated Hessian matrix (Holmes et al., 2012). In our study, we evaluated the model with the Shapiro-Wilk test (Royston, 1982) to identify the standardized residuals, checking the autocorrelations for the structure left in the residuals, which computes an estimate of the auto-correlation function (ACF) of the multivariate time series (Aryee et al., 2018). Due to the complexity of the multivariate models and the small sample sizes of the malaria cases, we used a $90 \%$ confidence interval to evaluate the MARSS simulated values in each cluster for estimation of the total trends and true patterns of malaria. Bootstrapping can be used to construct frequency condence intervals of the parameter estimates, and the MARSS model includes a semi-parametric bootstrap algorithm (Holmes et al., 2012) that affects the model residuals, i.e. the innovations. This approach uses the maximum-likelihood MARSS parameters to simulate data from which bootstrap parameter estimates are obtained. All test and data were analysed in R 3.3.2 software with MARSS packages (version 3.9) as used by (Holmes et al., 2012).

\section{Results}

The total number of the indigenous $P$. vivax cases in the study period from 2007 to 2009 showed nearly five times more indigenous cases in Tengchong than in any other county. The lowest case number ocurred in Changning County, where there was 10 cases as compared to none at all in 2009 . The number of malaria cases in the other three counties ranged from 0 to 32 within the three study years (Figure 2). A decreasing trend of indigenous $P$. vivax cases was observed in the Baoshan region in the period 2007-2009. Seasonal components varied among the five counties, such as an obvious periodicity pattern every year in Tengchong County, a peak from April to July occurred in Longning, Longyang and Shidian counties, while a climbing pattern with a peak from June to August was observed each year in Changning County. From the general survey of our research area, the map showed that more cases appeared in Tengchong, which is situated near the ChinaMyanmar border, while fewer cases happened in Changning situated further inside the Baoshan Prefecture and thus far from the border (Figure 1). Many foci cluster combinations were possible in our MARSS modelling. In this study, however, we only listed the top 20 best-fitted models according to the AICc values. The bestfitted model, which had the lowest AICc value (286.417), contained three hidden states (three foci clusters) (Table 1). In the models with the lowest AICc values, the malaria cases were all grouped into three clusters based on the hypotheses that all counties were sampled from different populations (Table 1). Tengchong represented cluster 1, Shidian was part of cluster 2, while the other three counties shared cluster 3 (Table 2). According to Table 2, each group had an unconstrained process error indicating that a 
group of counties within the same cluster had the same spatial pattern and that their dynamics were homogeneous. Meanwhile, the observation errors were found to be unequal with a diagonal matrix. Tengchong's observation error was lower (0.0135), while Changning had a largeer error $(0.3125)$ than all the other counties. As observed, the number of indigenous cases decreased in all of five counties, with negative reduction rates in all three clusters.
The reduction rate in cluster $3(-0.0537)$ decreased faster than that in the other two clusters suggesting the malaria infection is coming down in those counties. We evaluated the MARSS model by examination of the residual values, and comparison of the malaria indigenous cases between simulated and observed numbers. The simulation for the three clusters are shown in Figure 3. Tengchong was best-fitted to cluster 1 and cluster 2 did not extend beyond the

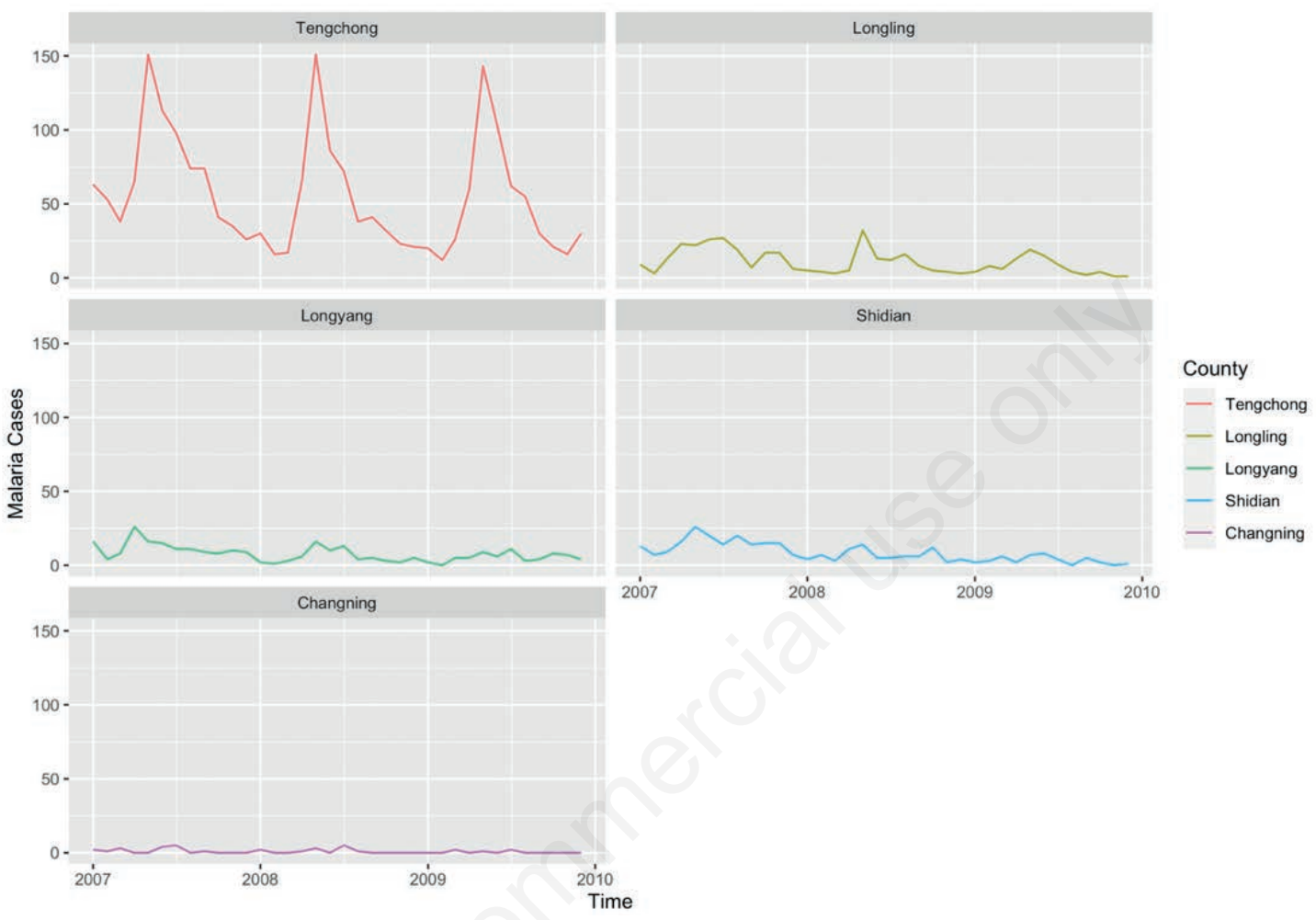

Figure 2. Monthly reported local Plasmodium vivax cases from five sites of Baoshan area, Yunnan Province, China.
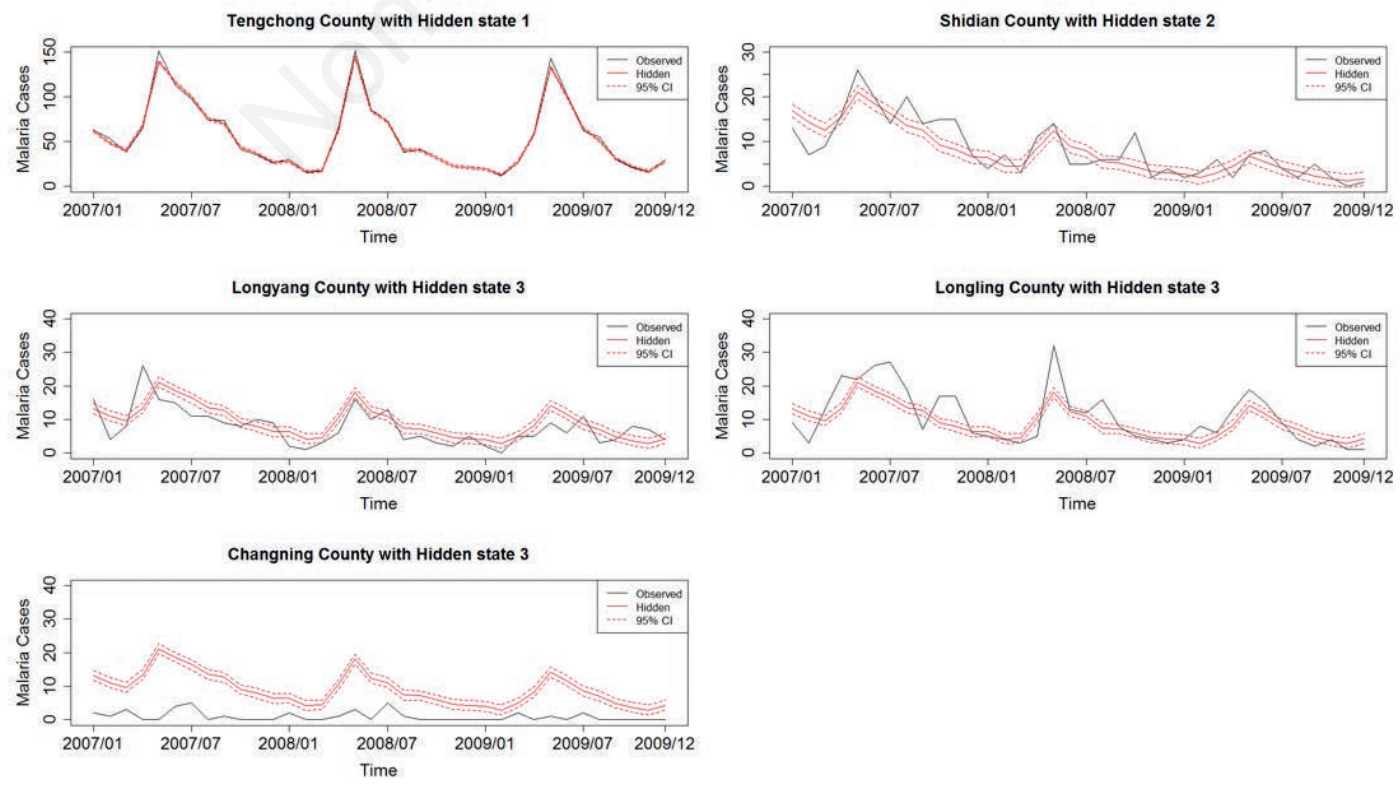

Figure 3. Estimation of best-fitted model for three clusters among the five sites and their $90 \%$ confidence intervals. 
borders of Shidian, while cluster 3 covered parts of all remaining three sites (Longling, Changning and Longyang). The fitted simulation in the three foci clusters was different from each other, with cluster 3 over-estimated in Changning which had the lowest number of indigenous cases. The $90 \%$ confidence interval in each foci cluster contained more than $90 \%$ of the malaria observation in the model simulations.

\section{Discussion}

This study attempted to apply a time series model to detect the clusters of foci by using malaria surveillance data in south-western
China near the Myanmar border in order to improve the surveillance sensitivity by targeted monitoring. China launched the malaria elimination programme in 2010 , and no indigenous cases were reproted in 2017 in the whole country based on passive surveillance (Feng et al., 2018), which is the main strategy to monitor malaria in China. However, there is a need to locate high-risk sites precisely and focus on the local level. For example, the reestablishment of high-risk malaria transmission in south-western China (Garros et al., 2006; Zhang et al., 2017; Yu et al., 2013; Li et al., 2015) remains to this day due to specific, climactic factors and proximity to endemic countries in South. Therefore, it is essential for local programme managers to make decisions and develop policies to further improve the sensitivity of the surveillance-

Table 1. The performance of 20 models ranked by Akaike's information criterion corrected based on different hypotheses with respect to the malaria cases.

\begin{tabular}{|c|c|c|c|c|c|c|c|c|c|}
\hline Model & $Q^{a}$ & $\mathbf{R}^{\mathrm{b}}$ & $\mathrm{U}^{\mathrm{c}}$ & $\log \operatorname{Lik}^{\mathrm{d}}$ & $\mathrm{AICc}^{\mathrm{e}}$ & $\mathrm{AIC}^{\mathrm{f}}$ & Parameters $^{g}$ & $\mathrm{M}^{\mathrm{h}}$ & Hypothesis $^{\mathrm{i}}$ \\
\hline 1 & 1 & 1 & 1 & -121.8338 & 286.4176 & 281.6676 & 19 & 3 & T-S-LYC \\
\hline 2 & 1 & 1 & 1 & -122.4028 & 287.5555 & 282.8055 & 19 & 3 & S-C-TLY \\
\hline 3 & 1 & 1 & 1 & -122.7177 & 288.1853 & 283.4353 & 19 & 3 & T-LS-YC \\
\hline 4 & 1 & 1 & 1 & -122.8298 & 288.4095 & 283.6595 & 19 & 3 & S-TL-YC \\
\hline 5 & 1 & 1 & 1 & -118.3369 & 292.4157 & 284.6738 & 24 & 4 & T-L-S-YC \\
\hline 6 & 1 & 1 & 1 & -127.4923 & 287.9114 & 284.9846 & 15 & 2 & SC-TLY \\
\hline 7 & 1 & 1 & 1 & -127.5118 & 287.9504 & 285.0236 & 15 & 2 & T-LYSC \\
\hline 8 & 1 & 1 & 1 & -127.5694 & 288.0657 & 285.1389 & 15 & 2 & S-TLYC \\
\hline 9 & 1 & 1 & 1 & -123.9823 & 290.7146 & 285.9646 & 19 & 3 & T-LY-SC \\
\hline 10 & 1 & 1 & 1 & -118.9946 & 293.7311 & 285.9891 & 24 & 4 & L-S-C-TY \\
\hline 11 & 1 & 1 & 1 & -124.0113 & 290.7725 & 286.0225 & 19 & 3 & L-S-TYC \\
\hline 12 & 1 & 1 & 1 & -124.1037 & 290.9574 & 286.2074 & 19 & 3 & C-TY-LS \\
\hline 13 & 1 & 1 & 1 & -124.2693 & 291.2885 & 286.5385 & 19 & 3 & T-C-LYS \\
\hline 14 & 1 & 2 & 1 & -128.5523 & 290.0315 & 287.1047 & 15 & 3 & S-C-TLY \\
\hline 15 & 1 & 1 & 1 & -128.6523 & 290.2313 & 287.3045 & 15 & 2 & TY-LSC \\
\hline 16 & 1 & 1 & 1 & -124.8893 & 292.5287 & 287.7787 & 19 & 3 & S-TY-LC \\
\hline 17 & 2 & 1 & 1 & -129.8895 & 290.3244 & 287.779 & 14 & 2 & LS-TYC \\
\hline 18 & 1 & 1 & 1 & -128.9868 & 290.9005 & 287.9737 & 15 & 2 & TL-YSC \\
\hline 19 & 1 & 1 & 1 & -125.0074 & 292.7648 & 288.0148 & 19 & 3 & L-TY-SC \\
\hline 20 & 2 & 1 & 1 & -129.2122 & 291.3511 & 288.4243 & 15 & 3 & S-TC-LY \\
\hline
\end{tabular}

Process errors with 1 for independent variances and 2 for the same variance; ${ }^{\mathrm{b}}$ observation errors with 1 for independent variances and 2 for same variance; ${ }^{\mathrm{c}}$ reduction rate with 1 for unequality among the data; ${ }^{\mathrm{d}}$ Loglikelihood of the data conditioned on the model parameters; ${ }^{\mathrm{e}}$ Corrected Akaike information criterion; ${ }^{\mathrm{A}}$ Akaike information criterion; ${ }^{\mathrm{g}}$ Number of parameters in the model; ${ }^{\mathrm{H}} \mathrm{Hypothesis}$ of hidden states among the five

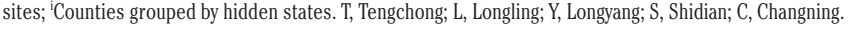

Table 2. Estimation of cluster growth rates, observation errors and process error in the five sites for the best-fitted model.

\begin{tabular}{|c|c|c|c|c|}
\hline Cluster & County & Reduction rate & Observation error & Process error \\
\hline 1 & Tengchong & $\begin{array}{c}-0.0223 \\
(-0.1783 ; 0.4858)\end{array}$ & $\begin{array}{c}0.0135 \\
(0.0048 ; 0.0910)\end{array}$ & $\begin{array}{c}0.2272 \\
(0.0771 ; 0.3261)\end{array}$ \\
\hline 2 & Shidian & $\begin{array}{c}-0.0537 \\
(-0.0909 ; 0.0165)\end{array}$ & $\begin{array}{c}0.1517 \\
(0.0884 ; 0.2320)\end{array}$ & $\begin{array}{c}0.0509 \\
(0.0003 ; 0.1010)\end{array}$ \\
\hline 3 & $\begin{array}{l}\text { Longling } \\
\text { Longyang } \\
\text { Changning }\end{array}$ & $\begin{array}{c}-0.0283 \\
(-0.0240 ; 0.0753)\end{array}$ & $\begin{array}{c}0.2229 \\
(0.0570,0.1298) \\
0.2074 \\
(0.1248 ; 0.3108) \\
0.3125 \\
(0.1771 ; 0.4858)\end{array}$ & $\begin{array}{c}0.0880 \\
(0.0218 ; 0.1455)\end{array}$ \\
\hline
\end{tabular}

Bootstrapped 90\% confidence interval for the reduction rate and the multivariate auto-regressive state-space model observation and process errors. 
response systems by targeting resources to identify foci of risk through sentinel monitoring (Hay et al., 2008).

Lately, no malaria cases have been reported from Baoshan Prefecture (Li et al., 2015), which was confirmed in the 2017 surveillance report. However, transmission varies from place to place and our results showed that the best-fitted model was the three-cluster pattern in Baoshan region, which implies that the five counties near the Myanmar border carry a high risk for continued transmission. It is obvious from Figure 1 that Tengchong near the border had a higher risk of malaria occurrence, something that has already been pointed out in previous studies (Dong et al., 2000). From the biological nature of malaria transmission, the geographical environment and climate in these areas represent intricate variables. In addition, vectors, such as Anopheles dirus and An. minimus do not respect political borders, which has recently been shown many times (Wang et al., 2015b; Chen et al. 2017; Zhang et al., 2018). Large numbers of An. minimus, An. dirus as well as An.sinensis populations have all been found in in Tengchong County (Dong, 2000; Wang et al., 2015b). Their habitats are associated with rice fields and various breeding sites. If those vectors find suitable ecological conditions during the transmission season, there is a strong possibility of re-introduction of malaria through imported $P$. vivax cases (Zhou et al., 2015a). Many reports show the cross-border activities play a major role in malaria transmission in the study region (Stoddard et al., 2009; Xu et al., 2016; Zhang et al., 2016; Shi et al., 2017), activities that also have varied from place to place and time to time, such as i) trade becoming more frequent in Tengchong County (Wang et al., 2015a; Li et al., 2016; Xu et al., 2016); ii) increasing numbers of imported cases enter this area continually (Yin et al., 2014); and iii) movements of people, e.g., labourer and farmers travelling across the border in particular months, leading to accumulated exposure opportunities (Yin et al., 2014). Reports show that Tengchong and Longling generally harbour the highest number of malaria cases, which are ascribed shared borders with Myanmar (Wang et al., 2016c) . Those living in other parts of Baoshan form relatively closed economic circumstances that result in fewer cross-border travel ( $\mathrm{Li}$ and Yang, 2015).

We find that our work based on MARSS modelling of time series matrix data provides real-time transmission information including the detection of risk spatio-temporal clusters. However, our cluster measures are different from China's "1-3-7" surveillance-response, which report malaria cases within 1 day, conducts a case investigation within 3 days and in-depth investigation within 7 days (Zhou et al., 2015b). This approach is based on cases reported in persons and sites, which means that if a case is identified, the "1-3-7" response is implemented limiting the number of cases in surrounding areas. Applying the MARSS model, however, spatiotemporal malaria clusters are instead searched at the regional level so that actions and surveillance can be focused at cluster regions when found. The whole region is thus considered and interventions can then be better directed, and the "1-3-7" surveillanceresponse more quickly targeted based on prepared resources and findings.

A number of studies have attempted to use biological data to define the structure of malaria transmission in the China-Myanmar border area. Firstly, Anopheles can easily transfer malaria from Myanmar to China or vise versa (Xu and Liu, 2016). The intensity depends mainly on density of the mosquitoes population and the local malaria prevalence, which is considered important transmission indicators in the border areas (Zhang et al., 2016). Work on genetic diversity of the mosquito population in Yunnan Province (Feng et al., 2017) has indicated that the vector gene flow is limited by geographical distance and geographical barriers (Liu et al., 2016). Secondly, the mosquito insecticide resistance varies from area to area, which contributes to diversity with repect to malaria incidence; however this resistance does not always overlap in sites at each sides of the border, something that may be explained by gene diversities of malaria populations that has so far been studied in larger areas, such as Yunnan Province as a whole (Wang et al., 2016b). Enlarged msquito genetics should be useful as the crossborder problems need to be solved. Further investigations of the different patterns of malaria transmission at the county level are thus warranted.

The incidence rate of malaria declined in Yunnan, but the rates fell differently from place to place. Such discrepancies across clusters in the study area are not surprising given the siuation near the Myanmar border compared to areas further away from the border (Figure 1). Although the overall annual number of indigenous cases have decreased gradually over the last few years, a pattern probably due to effective shared interventions implemented jointly in both China and Mynamar (Yin et al., 2014; Zhou et al., 2015b; Xu et al., 2017; Feng et al., 2018), we noticed differences of the reduction rate among the three clusters. However, even if the indigenous $P$. vivax cases in Tengchong tended down, there were still numerous cases in this county (Dong et al., 2000; Shi et al., 2015), which explains the good fitting results of the cluster simulations. Different locations had a different impact on malaria control, even if the total number of imported cases did not differ (Shi et al., 2014), which could be the cause of the difference in the reduction rate in these sites. Overall, the three clusters showed a high heterogeneity but shared a decreasing trend. To be successful, active surveillance should contain foci related to all the spatial patterns discovered in the Baoshan area.

The MARSS model could analyze the inside structures within the multiple time series based on various spatio-temporal clusters. Our modeling approach relied on the hypothesis that the reported malaria cases were from one population structure or more than one population. However, the situation gets progressively more complicated as the number of time series increase. The number of permutations and combinations of many clusters from multiple sites rapidly becomes too big for listing in a simple table. This should be considered when using MARSS model. To dispose of the problem of the permutations produced by the algorithm used, Ward et al. (2010) subdivided 13 sites into several sub-populations based on previous knowledge on the biology of different species. Although this approach can provide an explanation for the hypotheses, it can also miss some information. Furthermore, the integrated nested laplace approximation (INLA) method (Costa et al., 2011; Bivand et al., 2015), based on the spatial temporal Bayesian model, is another way of applying effective computation to predict the spatial dynamics of infection diseases. In our study, the malaria data were collected from many sites and identification of the clusters by time series data was our major purpose. So there is a need to update this state space model about permutation and combination algorithm to get a real understanding of the malaria transmission patterns. On the other hand, the surveillance-response systems used were based on the population level, which offers baseline access data. Application of MARSS modelling on smallarea surveillance data would produce more accurate active surveillance.

The limitations of the study are reflected in the following four 
aspects. The first challenge was that the data collection represented the county level and was based at only five restricted sites, which increased the risk of ending up with a comparatively large proportion of less precise data (Zhang et al., 2018). Second, from the simulation part, a low number of positive malaria data could result in a poor simulations leading to the over-estimated results from some counties. Third, the spatial distributions of infections emphasized that the risk area does not coincide with the administrative regions. Fourth, risk factors based on demography, environment and socioeconomy were not considered in the modelling.

The purpose of this study was to estimate malaria cluster structures about Baoshan area by MARSS, which produces spatial patterns from time series data. This could be a direct way to obtaining a general overview of malaria foci clusters supported by strong surveillance-response systems, as China is now approaching the stage of malaria elimination. Therefore, although the aforementioned four limitations exist, the MARSS model is still the best way to identifiy the clusters in this area, which in turn leads the delivery of health resources more efficiently (Feng et al., 2014).

\section{Conclusions}

Identification of focal clusters is important for the malaria elimination phase and critical for estimating the precise need for the health resources to be used for active surveillance. Applying the MARSS state-space model to several time series data at the county level made it possible to successfully estimate the reduction rate and the spatial patterns of indigenous malaria cases. Prevention of re-introduction of transmission into areas without any reported indigenous cases, requires processing data based on long-term monitoring by new methods. The approach investigated has provided a new, simple and direct tool to identify disease distributions capable of also predicting hidden states within trend parameters wich should help supporting long-term policies.

\section{References}

Anwar MY, Lewnard JA, Parikh S, Pitzer VE, 2016. Time series analysis of malaria in Afghanistan: using ARIMA models to predict future trends in incidence. Malar J. 15:566.

Aryee G, Kwarteng E, Essuman R, Nkansa Agyei A, Kudzawu S, Djagbletey R, Owusu Darkwa E, Forson A, 2018. Estimating the incidence of tuberculosis cases reported at a tertiary hospital in Ghana: a time series model approach. BMC Public Health 18:1292.

Bartocci E, Lió P, 2016. Computational modeling, formal analysis, and tools for systems biology. PLoS Comput Biol 12:e1004591.

Bivand R, Gómez-Rubio V, Rue H, 2015. Spatial data analysis with R-INLA with some extensions. J Stat Softw 63:1-31.

Costa AC, Codeço CT, Honório NA, Pereira GR, N Pinheiro CF, Nobre AA, 2015. Surveillance of dengue vectors using spatiotemporal Bayesian modeling. BMC Med Inform Decis Mak. 15:93.

Chen T, Zhang S, Zhou SS, Wang X, Luo C, Zeng X, Guo X, Lin Z, Tu H, Sun X, Zhou H, 2017. Receptivity to malaria in the China-Myanmar border in Yingjiang County, Yunnan Province, China. Malar J 16:478.
Cui L, Yan G, Sattabongkot J, Chen B, Cao Y, Fan Q, Parker D, Sirichaisinthop J, Su XZ, Yang H, Yang Z, Wang B, Zhou G, 2012. Challenges and prospects for malaria elimination in the Greater Mekong Subregion. Acta Trop 121:240-5.

Dong X, 2000. [The malaria vectors and their ecology in Yunnan Province.] Chin J Parasit Dis Contr. [Article in Chinese]. Available from: http://en.cnki.com.cn/Article_en/CJFDTOTAL-ZISC200002032.htm

Feng X, Huang L, Lin L, Yang M, Ma Y, 2017. Genetic diversity and population structure of the primary malaria vector Anopheles sinensis (Diptera: Culicidae) in China inferred by cox1 gene. Parasit Vectors 10:75. Erratum in: Parasit Vectors $10: 120$.

Feng XY, Xia ZG, Vong S, Yang WZ, Zhou SS, 2014. Surveillance and response to drive the national malaria elimination program. Adv Parasitol 86:81-108.

Feng J, Zhang L, Huang F, Yin JH, Tu H, Xia ZG, Zhou SS, Xiao N, Zhou XN, 2018. Ready for malaria elimination: zero indigenous case reported in the People's Republic of China. Malar J 17:315.

Garros C, Van Bortel W, Trung HD, Coosemans M, Manguin S, 2006. Review of the minimus complex of Anopheles, main malaria vector in Southeast Asia: from taxonomic issues to vector control strategies. Trop Med Int Health 11:102-14.

Gosling RD, Whittaker M, Gueye CS, Fullman N, Baquilod M, Kusriastuti R, Feachem RG, 2012. Malaria elimination gaining ground in the Asia Pacific. Malar J 11:346.

Hay SI, Smith DL, Snow RW, 2008. Measuring malaria endemicity from intense to interrupted transmission. Lancet Infect Dis 8:369-78

Hinrichsen RA, 2009. Population viability analysis for several populations using multivariate state-space models. Ecol Model 220:1197-202.

Holmes EE, Ward EJ, Wills K, 2012. MARSS: Multivariate autoregressive state-space models for analyzing time-series data. R J 4:11-9.

Hu T, Liu YB, Zhang SS, Xia ZG, Zhou SS, Yan J, Cao J, Feng ZC, 2016. Shrinking the malaria map in China: measuring the progress of the National Malaria Elimination Programme. Infect Dis Poverty 5:52.

Koc EK, Bozdogan H, 2015. Model selection in multivariate adaptive regression splines (MARSS) using information complexity as the fitness function. Machine Learn 101:35-58.

Liu H, Huang K, Liu S, Sun H, Lin K, Huang X, Chu J, Yang Z, 2016. Gene frequency and haplotype distribution of hemoglobin E among seven minority groups of Yunnan, China. Am J Hum Biol 28:927-31.

Li BF, Lin YX, Guo XR, Chen LF, Zhou DL, Yu GC, Zou J, Sun $\mathrm{XD}, 2015$. [Survey on malaria epidemics in China-Myanmar border area]. Zhongguo Ji Sheng Chong Xue Yu Ji Sheng Chong Bing Za Zhi 33:261-3 [Article in Chinese].

Li JQ, Yang HX, 2015. [Analysis of time distribution of malaria incidence in Baoshan City during 1990-2014.] Zhongguo Ji Sheng Chong Xue Yu Ji Sheng Chong Bing Za Zhi 33:309-11 [Article in Chinese].

Li S, Yin S, Wang J, Li X, Feng J, 2016. Shifting from control to elimination: analysis of malaria epidemiological characteristics in Tengchong County around China-Myanmar border, 2005-2014. Malar J 15:45.

Ministry of Health, China $(\mathrm{MoH}), 2010$. National Malaria Elimination Action Plan. Beijing, China. Available from: 
http://www.nhc.gov.cn/zwgk/wtwj/201304/15a4cc7a40b0452 191fe409590ca99d8.shtml

Reiner RC, Le Menach A, Kunene S, Ntshalintshali N, Hsiang MS, Perkins TA, Greenhouse B, Tatem AJ, Cohen JM, Smith DL, 2015. Mapping residual transmission for malaria elimination. Elife. 4:e09520.

Royston JP, 1982. An extension of Shapiro and Wilk's W test for normality to large samples. J Roy Stat Soc Series C (Appl Stat) 31:115-24.

Shi B, Liu J, Zhou XN, Yang GJ, 2014. Inferring Plasmodium vivax transmission networks from tempo-spatial surveillance data. PLoS Negl Trop Dis 8:e2682.

Shi B, Tan Q, Zhou XN, Liu J, 2015. Mining geographic variations of Plasmodium vivax for active surveillance: a case study in China. Malar J 14:216.

Shi B, Zheng J, Qiu H, Yang GJ, Xia S, Zhou XN, 2017. Risk assessment of malaria transmission at the border area of China and Myanmar. Infect Dis Poverty 6:108.

Stoddard ST, Morrison AC, Vazquez-Prokopec GM, Paz Soldan V, Kochel TJ, Kitron U, Elder JP, Scott TW, 2009. The role of human movement in the transmission of vector-borne pathogens. PLoS Negl Trop Dis 3:e481.

Tolimieri N, Holmes EE, Williams GD, Pacunski R, Lowry D, 2017. Population assessment using multivariate time-series analysis: A case study of rockfishes in Puget Sound. Ecol Evol 7:2846-60.

Valle D, Clark J, 2013. Improving the modeling of disease data from the government surveillance system: a case study on malaria in the Brazilian Amazon. PLoS Comput Biol 9:e1003312.

Wang D, He F, Maslov S, Gerstein M, 2016a. DREISS: using statespace models to infer the dynamics of gene expression driven by external and internal regulatory networks. PLoS Comput Biol 12:e1005146.

Wang D, Li S, Cheng Z, Xiao N, Cotter C, Hwang J, Li X, Yin S, Wang J, Bai L, Zheng Z, Wang S, 2015a. Transmission risk from imported plasmodium vivax malaria in the ChinaMyanmar border region. Emerg Infect Dis 21:1861-4.

Wang X, Yang L, Jiang T, Zhang B, Wang S, Wu X, Wang T, Li Y, Liu M, Peng Q, Zhang W, 2016b. Effects of a malaria elimination program: a retrospective study of 623 cases from 2008 to 2013 in a Chinese county hospital near the China-Myanmar border. Emerg Microbes Infect 5:e6.

Wang JZ, Yin SQ, Li SG, Li XS, Cai WB, Feng J, 2016c. [Evaluation of measures and achievements of malaria control in Tengchong City, Yunnan Province during 2010-2015.] Zhongguo Ji Sheng Chong Xue Yu Ji Sheng Chong Bing Za Zhi 34:435-8 [Article in Chinese].

Wang RB, Zhang QF, Zheng B, Xia ZG, Zhou SS, Tang LH, Gao Q, Wang LY, Wang RR, 2014. Transition from control to elimination: impact of the 10-year global fund project on malaria control and elimination in China. Adv Parasitol 86:289-318.

Wang Y, Zhong D, Cui L, Lee MC, Yang Z, Yan G, Zhou G, 2015b. Population dynamics and community structure of Anopheles mosquitoes along the China-Myanmar border. Parasit Vectors $8: 445$.

Wangdi K, Singhasivanon P, Silawan T, Lawpoolsri S, White NJ, Kaewkungwal J, 2010. Development of temporal modelling for forecasting and prediction of malaria infections using timeseries and ARIMAX analyses: a case study in endemic districts of Bhutan. Malar J 9:251.

Ward EJ, Chirakkal H, Gonzalezsuarez M, Auriolesgamboa D, Holmes EE, Gerber LR, 2010. Inferring spatial structure from time-series data: using multivariate state-space models to detect metapopulation structure of California sea lions in the Gulf of California, Mexico. J Appl Ecol 47:47-56.

Whittaker MA, Dean AJ, Chancellor A, 2014. Advocating for malaria elimination - learning from the successes of other infectious disease elimination programmes. Malar J 13:221.

World Health Organization (WHO), 2019. Malaria. Available from: http://www.who.int/mediacentre/factsheets/fs094/en/

Xu JW, Li JJ, Guo HP, Pu SW, Li SM, Wang RH, Liu H, Wang WJ, 2017. Malaria from hyperendemicity to elimination in Hekou County on China-Vietnam border: an ecological study. Malar J 16:66.

Xu JW, Li Y, Yang HL, Zhang J, Zhang ZX, Yang YM, Zhou HN, Havumaki J, Li HX, Liu H, Zhou H, Xie XY, Dong JX, Zhang Y, Sun XY, Li B, Li JY, Tian YH, Wang PY, Li BF, 2016. Malaria control along China-Myanmar Border during 20072013: an integrated impact evaluation. Infect Dis Poverty 5:75.

Xu JW, Liu H, 2016. The relationship of malaria between Chinese side and Myanmar's five special regions along ChinaMyanmar border: a linear regression analysis. Malar J 15:368.

Yin JH, Zhou SS, Xia ZG, Wang RB, Qian YJ, Yang WZ, Zhou $\mathrm{XN}$, 2014. Historical patterns of malaria transmission in China. Adv Parasitol 86:1-19.

Yu G, Yan G, Zhang N, Zhong D, Wang Y, He Z, Yan Z, Fu W, Yang F, Chen B, 2013. The Anopheles community and the role of Anopheles minimus on malaria transmission on the ChinaMyanmar border. Parasit Vectors 6:264.

Zhang S, Guo S, Feng X, Afelt A, Frutos R, Zhou S, 2017. Manguin S. Anopheles vectors in mainland China while approaching malaria elimination. Trends Parasitol 33:889-900.

Zhang Q, Sun J, Zhang Z, Geng Q, Lai S, Hu W, Clements AC, Li Z, 2016. Risk assessment of malaria in land border regions of China in the context of malaria elimination. Malar J 15:546.

Zhang SS, Zhou SS, Zhou ZB, Chen TM, Wang XZ, Shi WQ, Jiang WK, Li JL, Zhou XN, Frutos R, Manguin S, Afelt A, 2018. Monitoring of malaria vectors at the China-Myanmar border while approaching malaria elimination. Parasit Vectors 11:511.

Zhou P, Wang D, Hwang J, Bennett A, Sturrock HJ, Ma A, Huang J, Xia Z, Feng X, Wang J, 2015a. Spatial-temporal variation and primary ecological drivers of Anopheles sinensis human biting rates in malaria epidemic-prone regions of China. PLoS One 10:e0116932.

Zhou SS, Wang Y, Li Y, 2011. [Malaria situation in the People's Republic of China in 2010.] Zhongguo Ji Sheng Chong Xue Yu Ji Sheng Chong Bing Za Zhi 29:401-3. [Article in Chinese].

Zhou SS, Zhang SS, Zhang L, Rietveld AE, Ramsay AR, Zachariah R, Bissell K, Van den Bergh R, Xia ZG, Zhou XN, Cibulskis RE, 2015b. China's 1-3-7 surveillance and response strategy for malaria elimination: Is case reporting, investigation and foci response happening according to plan? Infect Dis Poverty 4:55. 